\title{
EXTREMAL ALGEBRAIC CONNECTIVITIES OF CERTAIN CATERPILLAR CLASSES AND SYMMETRIC CATERPILLARS*
}

\author{
OSCAR ROJO ${ }^{\dagger}$, LUIS MEDINA ${ }^{\dagger}$, NAIR M. M. DE ABREU ${ }^{\ddagger}$, AND CLAUDIA JUSTEL ${ }^{\S}$
}

Abstract. A caterpillar is a tree in which the removal of all pendant vertices makes it a path. Let $d \geq 3$ and $n \geq 6$ be given. Let $P_{d-1}$ be the path of $d-1$ vertices and $S_{p}$ be the star of $p+1$ vertices. Let $\mathbf{p}=\left[p_{1}, p_{2}, \ldots, p_{d-1}\right]$ such that $p_{1} \geq 1, p_{2} \geq 1, \ldots, p_{d-1} \geq 1$. Let $C(\mathbf{p})$ be the caterpillar obtained from the stars $S_{p_{1}}, S_{p_{2}}, \ldots, S_{p_{d-1}}$ and the path $P_{d-1}$ by identifying the root of $S_{p_{i}}$ with the $i$-vertex of $P_{d-1}$. Let $n>2(d-1)$ be given. Let

$$
\mathcal{C}=\left\{C(\mathbf{p}): p_{1}+p_{2}+\ldots+p_{d-1}=n-d+1\right\}
$$

and

$$
\mathcal{S}=\left\{C(\mathbf{p}) \in \mathcal{C}: p_{j}=p_{d-j}, j=1,2, \cdots,\left\lfloor\frac{d-1}{2}\right\rfloor\right\} .
$$

In this paper, the caterpillars in $\mathcal{C}$ and in $\mathcal{S}$ having the maximum and the minimum algebraic connectivity are found. Moreover, the algebraic connectivity of a caterpillar in $\mathcal{S}$ as the smallest eigenvalue of a $2 \times 2$ - block tridiagonal matrix of order $2 s \times 2 s$ if $d=2 s+1$ or $d=2 s+2$ is characterized.

Key words. Laplacian matrix, Algebraic connectivity, Caterpillar, Bottleneck matrices, Perron branches, Characteristic vertices.

AMS subject classifications. 05C50, 15A48, $05 \mathrm{C} 05$.

1. Introduction. Let $G=(V, E)$ be a simple undirected graph on $n$ vertices. The Laplacian matrix of $G$ is the $n \times n$ matrix $L(G)=D(G)-A(G)$ where $A(G)$ is the adjacency matrix and $D(G)$ is the diagonal matrix of vertex degrees. It is known that $L(G)$ is a positive semidefinite matrix and $(0, \mathbf{e})$ is an eigenpair of $L(G)$ where $\mathbf{e}$ is the all ones vector. In [12], some of the many results known for Laplacian matrices are given. Fiedler [5] proves that $G$ is a connected graph if and only if the second smallest Laplacian eigenvalue is positive. This eigenvalue is called the algebraic connectivity of $G$, it is denoted by $a(G)$ and any of its corresponding eigenvectors

${ }^{*}$ Received by the editors October 5, 2009. Accepted for publication February 15, 2010. Handling Editor: Michael Neumann.

$\dagger$ Department of Mathematics, Universidad Católica del Norte, Antofagasta, Chile. Work supported by Project Fondecyt 1070537, Chile (orojo@ucn.cl). Part of this research was conducted while O. Rojo was a visitor at the Centro de Modelamiento Matemático, Universidad de Chile, Santiago, Chile.

${ }^{\ddagger}$ Universidad Federal de Rio de Janeiro, Rio de Janeiro, Brazil. Work supported by CNPq 300563/94-9, Brazil.

$\S$ Instituto Militar de Engenharia, Rio de Janeiro, Brazil. 
is called a Fiedler vector. In [3], a survey of old and new results on the algebraic connectivity of graphs is given.

We recall that a tree is a connected acyclic graph. Let us denote by $P_{n}$ a path of $n$ vertices and by $S_{p}$ a star of $p+1$ vertices. Let $n$ and $d$ be fixed. Let $T(a, b, d)$ be the tree with $n$ vertices and diameter $d$ obtained from the path $P_{d-1}$ and the stars $S_{a}$ and $S_{b}$ by identifying the pendant vertices in $P_{d-1}$ with the centers of the stars. We may consider $a \leq b$. Observe that $b=n-a-(d-1)$. Then $1 \leq a \leq \frac{1}{2}(n-d+1)$.

The problem of ordering trees by algebraic connectivity is an active area of research. The next theorem due to Grone and Merris solves the problem for trees of order $n$ and diameter $d=3$.

TheOREM 1.1. [7] The algebraic connectivity of $T(a, b, 3), b=n-a-2$, is the unique Laplacian eigenvalue less than 1 and it is a strictly decreasing function for $1 \leq a \leq \frac{1}{2}(n-2)$.

Important contributions to the problem for trees of order $n$ and diameter $d=4$ are due to X-D Zhang [19, 2007]. Shao et al. [15, 2008] determine the first four trees of order $n \geq 9$ with the smallest algebraic connectivity. Yuan et al. [17, 2007] introduce six classes of trees with $n$ vertices and determine the ordering of those trees by this spectral invariant. Zhang and Liu $[18,2008]$ find the largest twelve values of algebraic connectivity of trees in a set of trees on $2 k+1$ vertices with nearly perfect matching. In [4], Fallat and Kirkland find the tree that minimizes and the tree that maximizes the algebraic connectivity among the trees on $n$ vertices and diameter $d$ :

Theorem 1.2. [4, Theorem 3.2] Among all trees on $n$ vertices with fixed diameter $d$, the minimum algebraic connectivity is attained by the tree

$$
T\left(\left\lfloor\frac{n-d+1}{2}\right\rfloor,\left\lceil\frac{n-d+1}{2}\right\rceil, d\right) .
$$

Theorem 1.3. [4, Theorem 3.8] Among all trees on $n$ vertices with fixed diameter $d$, the maximum algebraic connectivity is attained by the tree $P_{d, n-d}$ obtained from the path $P_{d}$ on vertices $1,2, \ldots, d$ and the star $S_{n-d}$ identifying the root of $S_{n-d}$ with the vertex $\left\lceil\frac{d+1}{2}\right\rceil$ of the path $P_{d}$.

A caterpillar is a tree in which the removal of all pendant vertices makes it a path. Let $n>2(d-1)$ be given with $d \geq 3$. Let $\mathbf{p}=\left[p_{1}, p_{2}, \ldots, p_{d-1}\right]$. with $p_{1} \geq 1, p_{2} \geq 1, \ldots, p_{d-1} \geq 1$. Let $C(\mathbf{p})$ be the caterpillar obtained from the stars $S_{p_{1}}, S_{p_{2}}, \ldots, S_{p_{d-1}}$ and the path $P_{d-1}$ by identifying the root of $S_{p_{i}}$ with the $i$-vertex of $P_{d-1}$. 
Let

$$
\mathcal{C}=\left\{C(\mathbf{p}): p_{1}+p_{2}+\ldots+p_{d-1}=n-d+1\right\}
$$

and

$$
\mathcal{S}=\left\{C(\mathbf{p}) \in \mathcal{C}: p_{j}=p_{d-j}, j=1,2, \ldots,\left\lfloor\frac{d-1}{2}\right\rfloor\right\} .
$$

Clearly any caterpillar in $\mathcal{C}$ is a caterpillar on $n$ vertices with diameter $d$.

In [2], the authors prove that the caterpillars are the trees having minimum algebraic connectivity among all trees with a given degree sequence.

In this paper, we find the caterpillars in $\mathcal{C}$ and in $\mathcal{S}$ having the maximum and the minimum algebraic connectivity. Moreover, we characterize the algebraic connectivity of a caterpillar in $\mathcal{S}$ as the smallest eigenvalue of a $2 \times 2$ - block tridiagonal matrix of order $2 s \times 2 s$ if $d=2 s+1$ or $d=2 s+2$.

2. Basic tools. In this Section, we present some results on the algebraic connectivity of trees which are the basic tools of this paper.

Lemma 2.1. [8, Corollary 4.2] Let $v$ be a pendant vertex of the graph $\widetilde{G}$. Let $G$ be the graph obtained from $\widetilde{G}$ by removing $v$ and its edge. Then the eigenvalues of $L(G)$ interlace the eigenvalues of $L(\widetilde{G})$.

From Lemma 2.1, it follows

Corollary 2.2. Let $T$ be a subtree of the tree $\widetilde{T}$. Then

$$
a(\widetilde{T}) \leq a(T) .
$$

For the rest of this Section, $T$ is a tree on vertices $1,2, \ldots, n$ and

$$
\mathbf{f}=\left[\begin{array}{llllll}
f_{1} & f_{2} & \cdots & \cdots & f_{n-1} & f_{n}
\end{array}\right]^{T} .
$$

TheOrem 2.3. [6] If $\mathbf{f}$ is a Fiedler vector then exactly one of the following two cases occurs:

(A) No entry of $\mathbf{f}$ is 0 . In this case, there is a unique pair of vertices $i$ and $j$ such that $i$ and $j$ are adjacent with $f_{i}>0$ and $f_{j}<0$.

(B) Some entry of $\mathbf{f}$ is 0 . In this case, the subgraph induced by the vertices corresponding to zeros in $\mathbf{f}$ is a connected subgraph. Moreover, there is a unique vertex $k$ such that $f_{k}=0$ and $k$ is adjacent to a vertex $i$ with $f_{i} \neq 0$. 
In [11], Merris proves that the occurrence of (A) or (B) does not depend on the Fiedler vector.

Definition 2.4. 1. $T$ is said Type $I I$ if $(A)$ holds and the vertices $i$ and $j$ are called the characteristic vertices of $T$. The edge defined by $i$ and $j$ is called the characteristic edge of $T$.

2. The tree $T$ is said Type $I$ if $(B)$ holds and the vertex $k$ is called the characteristic vertex of $T$.

3. Let $m$ be a vertex of the tree $T$. A branch of $T$ at $m$ is any of the connected component of $T-m$ obtained from $T$ removing the vertex $m$ and the edges incident with $m$.

Lemma 2.5. [10] Let $L_{k}$ be the principal submatrix of the Laplacian matrix $L(T)$ obtained by deleting the $k$-row and $k$-column from $L(T)$. Then the $(i, j)$-entry of $L_{k}^{-1}$ is equal to the number of edges of $T$ which are on both the path from vertex $i$ to vertex $k$ and the path from vertex $j$ to vertex $k$.

EXAMPLE 2.6. Let $T$ be the caterpillar below

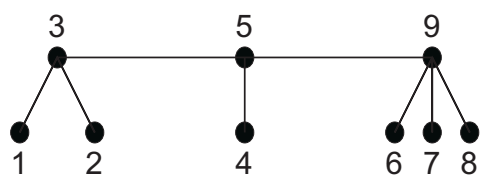

Then

$$
L_{5}^{-1}=\left[\begin{array}{cccccccc}
2 & 1 & 1 & 0 & 0 & 0 & 0 & 0 \\
1 & 2 & 1 & 0 & 0 & 0 & 0 & 0 \\
1 & 1 & 1 & 0 & 0 & 0 & 0 & 0 \\
0 & 0 & 0 & 1 & 0 & 0 & 0 & 0 \\
0 & 0 & 0 & 0 & 2 & 1 & 1 & 1 \\
0 & 0 & 0 & 0 & 1 & 2 & 1 & 1 \\
0 & 0 & 0 & 0 & 1 & 1 & 2 & 1 \\
0 & 0 & 0 & 0 & 1 & 1 & 1 & 1
\end{array}\right]
$$

Clearly the $(i, j)$ entry of $L_{k}^{-1}$ is positive if and only if the vertices $i$ and $j$ are in the same branch of $T$ at the vertex $k$. Then there is a labeling of the vertices of $T$ such that $L_{k}^{-1}$ is similar to a block diagonal matrix in which the number of diagonal blocks is the degree of the vertex $k$. Moreover, each diagonal block is a positive matrix corresponding to a unique branch at $k$. This matrix is called the bottleneck matrix for that branch at $k$. In Example 2.6, the bottleneck matrices for the branches at the 
vertex 5 are the matrices

$$
\left[\begin{array}{lll}
2 & 1 & 1 \\
1 & 2 & 1 \\
1 & 1 & 1
\end{array}\right],[1],\left[\begin{array}{llll}
2 & 1 & 1 & 1 \\
1 & 2 & 1 & 1 \\
1 & 1 & 2 & 1 \\
1 & 1 & 1 & 1
\end{array}\right]
$$

From the Perron-Frobenius Theory for nonnegative matrices, it follows that each bottleneck matrix has a simple eigenvalue equal to its spectral radius, its Perron root.

Definition 2.7. The Perron root of the bottleneck matrix of a branch is called the Perron root of the branch. A branch is called a Perron branch if its Perron root is equal to the spectral radius of $L_{k}^{-1}$.

The notion of Perron branches was first introduced by Kirkland, Neumann and Shader [10].

Let $\rho(A)$ be the spectral radius of a matrix $A$ and let $\mathbf{e}$ be the all ones vector of the appropriate order.

TheOREM 2.8. [10] Let $i$ and $j$ be adjacent vertices of $T$. Then $T$ is Type II tree with characteristic vertices $i$ and $j$ if and only if there exists $0<\gamma<1$ such that

$$
\rho\left(M-\gamma \mathbf{e} \mathbf{e}^{T}\right)=\rho\left(\widehat{M}-(1-\gamma) \mathbf{e e}^{T}\right)
$$

where $M$ is the bottleneck matrix of the branch at $j$ containing $i$ and $\widehat{M}$ is the bottleneck matrix of the branch at $i$ containing $j$. Moreover, if this condition holds then

$$
\frac{1}{a(T)}=\rho\left(M-\gamma \mathbf{e e}^{T}\right)=\rho\left(\widehat{M}-(1-\gamma) \mathbf{e e}^{T}\right)
$$

and any eigenvector of $L(T)$ corresponding to a $(T)$ can be permuted such that it has the form $\left[\begin{array}{c}-\mathbf{f}_{1} \\ \mathbf{f}_{2}\end{array}\right]$ where $\mathbf{f}_{1}$ is a Perron vector for $M-\gamma \mathbf{e e}^{T}$ and $\mathbf{f}_{2}$ is a Perron vector for $\widehat{M}-(1-\gamma) \mathbf{e} \mathbf{e}^{T}$ and $\mathbf{e}^{T} \mathbf{f}_{1}=\mathbf{e}^{T} \mathbf{f}_{2}$.

COROllary 2.9. $T$ is a Type II tree with characteristic vertices $i$ and $j$ if and only if (1) the vertices $i$ and $j$ are adjacent, (2) the branch at $i$ containing the vertex $j$ is the unique Perron branch at $i$, and (3) the branch at $j$ containing the vertex $i$ is the unique Perron branch at $j$.

THEOREM 2.10. [10] $T$ is a Type I tree with characteristic vertex $k$ if and only if there are two or more Perron branches of $T$ at $k$. In this case,

$$
a(T)=\frac{1}{\rho\left(L_{k}^{-1}\right)}
$$


and if $\mathbf{f}$ is an eigenvector corresponding to a $(T)$ then $\mathbf{f}$ can be permuted such that each of the resulting nonzero subvectors is a Perron vector for the bottleneck matrix of a Perron branch at $k$.

Corollary 2.11. T is a Type I tree if and only if there is exactly one vertex at which there are two or more Perron branches. $T$ is a Type II tree if and only if at each vertex there is unique Perron branch.

\section{Extremal algebraic connectivities.}

3.1. Characteristic vertices of caterpillars and their Perron branches. Let $v_{1}, v_{2}, \ldots, v_{d-1}$ be the vertices of the path $P_{d-1}$. We observe that for any tree, no pendant vertex is a characteristic vertex. From this fact and Theorem 2.8, it follows

Lemma 3.1. If $C_{t} \in \mathcal{C}$ is a Type II tree and $v_{i}$ and $v_{i+1}$ are its characteristic vertices then

$$
\frac{1}{a\left(C_{t}\right)}=\rho\left(M_{t}-\gamma_{t} \mathbf{e e}^{T}\right)=\rho\left(\widehat{M}_{t}-\left(1-\gamma_{t}\right) \mathbf{e e}^{T}\right)
$$

where $M_{t}$ is the bottleneck matrix of the Perron branch $B_{t}$ at $v_{i+1}$ containing the vertex $v_{i}$ and $\widehat{M}_{t}$ is the bottleneck matrix of the Perron branch $\widehat{B}_{t}$ at $v_{i}$ containing the vertex $v_{i+1}$. From Theorem 2.10, it follows

Lemma 3.2. If $C_{t} \in \mathcal{C}$ is a Type $I$ tree and $v_{i}$ is its characteristic vertex then

$$
\frac{1}{a\left(C_{t}\right)}=\rho\left(M_{t}\right)=\rho\left(\widehat{M}_{t}\right)
$$

where $M_{t}$ and $\widehat{M}_{t}$ are the bottleneck matrices of $B_{t}$ and $\widehat{B}_{t}$, respectively, in which $B_{t}$ is the branch containing the vertex $v_{1}$ and $\widehat{B}_{t}$ is the branch containing the vertex $v_{d-1}$.

3.2. Maximum algebraic connectivity. We first consider the case of $d$ even. This case is already studied in [1, Theorem 9]. Here, we give a different proof.

TheOREM 3.3. If $d=2 s+2$ then among all trees in $\mathcal{C}$ the maximum algebraic connectivity is attained by the caterpillar $C(\mathbf{q})$ where $q_{i}=1$ for all $i \neq s+1$ and $q_{s+1}=n-2 d+3$.

Proof. Observe that $C(\mathbf{q}) \in \mathcal{C}$. Let $\mathbf{e}$ be the all ones vector of order $d-1$. Let $C_{1}=C(\mathbf{p}) \in \mathcal{C}, C_{2}=C(\mathbf{e})$ and $C_{3}=C(\mathbf{q})$. Clearly $C_{2}$ is a subtree of $C_{1}$. Applying Corollary 2.2, $a\left(C_{1}\right) \leq a\left(C_{2}\right)$. Moreover, it is clear that $C_{2}$ and $C_{3}$ are both trees of Type $I$ at the same characteristic vertex $v_{s}$ having isomorphic Perron branches. Hence, applying Theorem 2.10, $a\left(C_{2}\right)=a\left(C_{3}\right)$. Therefore $a\left(C_{1}\right) \leq a\left(C_{3}\right)$. 
For the case of odd $d$, to get the caterpillar in $\mathcal{C}$ with the maximum algebraic connectivity is not easy as in Theorem 3.3.

At this point, it is convenient to recall that if $A$ is an irreducible nonnegative (in particular, positive) matrix then $\rho(A)$ strictly increases when any entry of $A$ strictly increases [16, Theorem 2.1.].

Lemma 3.4. Let $d=2 s+1$. Let

$$
\begin{aligned}
C & =C\left(p_{1}, \ldots, p_{s}, p_{s+1}, \ldots, p_{2 s}\right) \in \mathcal{C} \\
\widetilde{p}_{s} & =\sum_{l=1}^{s} p_{l}-s+1 \\
\widetilde{p}_{s+1} & =\sum_{l=s+1}^{2 s} p_{l}-s+1 .
\end{aligned}
$$

Then

$$
a(C) \leq a(\widetilde{C})
$$

where

$$
\widetilde{C}=C\left(1, \ldots, 1, \widetilde{p}_{s}, \widetilde{p}_{s+1}, 1, \ldots, 1\right) .
$$

The equality holds if and only if $C=\widetilde{C}$.

Proof. Let $C_{1}=C, C_{3}=\widetilde{C}$ and

$$
C_{2}=C\left(1, \ldots, 1, \widetilde{p}_{s}, p_{s+1}, \ldots, p_{2 s}\right) .
$$

Clearly $C_{2}, C_{3} \in \mathcal{C}$ and $s=\frac{1}{2}(d-1)$. Let $v_{1}, \ldots, v_{s}, v_{s+1}, \ldots, v_{2 s}$ be the vertices of the path $P_{2 s}$. We first prove that

$$
a\left(C_{1}\right) \leq a\left(C_{2}\right)
$$

with equality if and only if $C_{1}=C_{2}$.

If $C_{1}=C_{2}$ then $a\left(C_{1}\right)=a\left(C_{2}\right)$. Suppose $C_{1} \neq C_{2}$. Then $p_{t}>1$ for some $1 \leq t \leq s-1$. Four cases can occur. In each case, the vertices of $C_{1}$ and $C_{2}$ are labeled appropriately to compare the entries of the corresponding bottleneck matrices in $(3.1)$ or $(3.2)$.

Case $C_{1}$ and $C_{2}$ are both of Type $I$ tree. Let $w_{1}$ and $w_{2}$ be the characteristic vertices of $C_{1}$ and $C_{2}$, respectively. Then

$$
\frac{1}{a\left(C_{1}\right)}=\rho\left(M_{1}\right)=\rho\left(\widehat{M}_{1}\right) \text { and } \frac{1}{a\left(C_{2}\right)}=\rho\left(M_{2}\right)=\rho\left(\widehat{M}_{2}\right) \text {. }
$$


We have $w_{1}=v_{l}$ for some $l$ and $w_{2}=v_{k}$ for some $k \geq s+1$. If $l<k, \widehat{B}_{2}$ is isomorphic to a proper subtree of $\widehat{B}_{1}$ and then $\widehat{M}_{1}(p, q)-\widehat{M}_{2}(p, q)>1$ for each $(p, q)$. Hence

$$
\frac{1}{a\left(C_{1}\right)}=\rho\left(\widehat{M}_{1}\right)>\rho\left(\widehat{M}_{2}\right)=\frac{1}{a\left(C_{2}\right)} .
$$

If $l \geq k$, since $p_{t}>1$ for some $1 \leq t \leq s-1$, we have $M_{1}(p, q)-M_{2}(p, q) \geq 0$ for each $(p, q)$ with strict inequality in at least one entry. Hence

$$
\frac{1}{a\left(C_{1}\right)}=\rho\left(M_{1}\right)>\rho\left(M_{2}\right)=\frac{1}{a\left(C_{2}\right)} .
$$

Case $C_{1}$ is a Type I tree and $C_{2}$ is a Type II tree. Let $v_{l}$ be the characteristic vertex of $C_{1}$. Let $v_{i}$ and $v_{i+1}$ be the characteristic vertices of $C_{2}$. There is $0<\gamma_{2}<1$ such that

$$
\frac{1}{a\left(C_{2}\right)}=\rho\left(M_{2}-\gamma_{2} \mathbf{e e}^{T}\right)=\rho\left(\widehat{M}_{2}-\left(1-\gamma_{2}\right) \mathbf{e e}^{T}\right) .
$$

Clearly, $i \geq s$. If $i=l$ then

$$
\frac{1}{a\left(C_{1}\right)}=\rho\left(\widehat{M}_{1}\right)=\rho\left(\widehat{M}_{2}\right)>\rho\left(\widehat{M}_{2}-\left(1-\gamma_{2}\right) \mathbf{e e}^{T}\right)=\frac{1}{a\left(C_{2}\right)} .
$$

If $i>l$ then $\widehat{B}_{2}$ is isomorphic to a proper subtree of $\widehat{B}_{1}$, thus $\widehat{M}_{1}(p, q)-\widehat{M}_{2}(p, q)>1$ for each $(p, q)$ and hence

$$
\frac{1}{a\left(C_{1}\right)}=\rho\left(\widehat{M}_{1}\right)>\rho\left(\widehat{M}_{2}\right)>\rho\left(\widehat{M}_{2}-\left(1-\gamma_{2}\right) \mathbf{e e}^{T}\right)=\frac{1}{a\left(C_{2}\right)} .
$$

If $i<l$, since $p_{t}>1$ for some $1 \leq t \leq s-1$, we have $M_{1}(p, q)-M_{2}(p, q) \geq 0$ for each $(p, q)$ with strict inequality in at least one entry. Hence

$$
\frac{1}{a\left(C_{1}\right)}=\rho\left(M_{1}\right)>\rho\left(M_{2}\right)>\rho\left(M_{2}-\gamma_{2} \mathbf{e e}^{T}\right)=\frac{1}{a\left(C_{2}\right)} .
$$

Case $C_{1}$ is a Type II tree and $C_{2}$ is a Type I tree. Let $v_{k}$ be the characteristic vertex of $C_{2}$. Then $k \geq s+1$. Let $v_{i}$ and $v_{i+1}$ be the characteristic vertices of $C_{1}$. There is $0<\gamma_{1}<1$ such that

$$
\frac{1}{a\left(C_{1}\right)}=\rho\left(M_{1}-\gamma_{1} \mathbf{e e}^{T}\right)=\rho\left(\widehat{M}_{1}-\left(1-\gamma_{1}\right) \mathbf{e e}^{T}\right) .
$$

If $i<k$ then the branch $\widehat{B}_{1}$ contains the vertex $v_{k}$ and an isomorphic copy of $\widehat{B}_{2}$, thus $\widehat{M}_{1}(p, q)-\widehat{M}_{2}(p, q) \geq 1$ for each $(p, q)$. Hence

$$
\frac{1}{a\left(C_{1}\right)}=\rho\left(\widehat{M}_{1}-\left(1-\gamma_{1}\right) \mathbf{e e}^{T}\right)>\rho\left(\widehat{M}_{2}\right)=\frac{1}{a\left(C_{2}\right)}
$$


If $i \geq k$ then $M_{1}(p, q)-M_{2}(p, q) \geq 1$ for each $(p, q)$. Hence

$$
\frac{1}{a\left(C_{1}\right)}=\rho\left(M_{1}-\gamma_{1} \mathbf{e e}^{T}\right)>\rho\left(M_{2}\right)=\frac{1}{a\left(C_{2}\right)} .
$$

Case $C_{1}$ and $C_{2}$ are both of Type II. Let $v_{j}$ and $v_{j+1}$ the characteristic vertices of $C_{1}$. Let $v_{i}$ and $v_{i+1}$ be the characteristic vertices of $C_{2}$. There are $0<\gamma_{1}<1$ and $0<\gamma_{2}<1$ such that

$$
\begin{aligned}
& \frac{1}{a\left(C_{1}\right)}=\rho\left(M_{1}-\gamma_{1} \mathbf{e e}^{T}\right)=\rho\left(\widehat{M}_{1}-\left(1-\gamma_{1}\right) \mathbf{e e}^{T}\right) \\
& \frac{1}{a\left(C_{2}\right)}=\rho\left(M_{2}-\gamma_{2} \mathbf{e e}^{T}\right)=\rho\left(\widehat{M}_{2}-\left(1-\gamma_{2}\right) \mathbf{e e}^{T}\right) .
\end{aligned}
$$

As before, $i \geq s$. If $j<i, \widehat{M}_{1}(p, q)-\widehat{M}_{2}(p, p) \geq 1$ for each $(p, q)$. Then

$$
\begin{aligned}
\frac{1}{a\left(C_{1}\right)} & =\rho\left(\widehat{M}_{1}-\left(1-\gamma_{1}\right) \mathbf{e e}^{T}\right)>\rho\left(\widehat{M}_{2}\right) \\
& >\rho\left(\widehat{M}_{2}-\left(1-\gamma_{2}\right) \mathbf{e e}^{T}\right)=\frac{1}{a\left(C_{2}\right)} .
\end{aligned}
$$

If $j>i, M_{1}(p, q)-M_{2}(p, p) \geq 1$ for each $(p, q)$. Then

$$
\frac{1}{a\left(C_{1}\right)}=\rho\left(M_{1}-\gamma_{1} \mathbf{e e}^{T}\right)>\rho\left(M_{2}\right)>\rho\left(M_{2}-\gamma_{2} \mathbf{e e}^{T}\right)=\frac{1}{a\left(C_{2}\right)} .
$$

If $j=i$, then $\widehat{M}_{1}=\widehat{M}_{2}$ and $M_{1} \geq M_{2}$. Moreover, since $p_{t}>1$ for some $1 \leq t \leq s-1$, we have $M_{1}(p, q)>M_{2}(p, q)$ for some $(p, q)$. If $\gamma_{1}=\gamma_{2}$ then

$$
\frac{1}{a\left(C_{1}\right)}=\rho\left(\widehat{M}_{1}-\left(1-\gamma_{1}\right) \mathbf{e e}^{T}\right)=\rho\left(\widehat{M}_{2}-\left(1-\gamma_{2}\right) \mathbf{e e}^{T}\right)=\frac{1}{a\left(C_{2}\right)} .
$$

Moreover

$$
\frac{1}{a\left(C_{1}\right)}=\rho\left(M_{1}-\gamma_{1} \mathbf{e e}^{T}\right)>\rho\left(M_{2}-\gamma_{2} \mathbf{e e}^{T}\right)=\frac{1}{a\left(C_{2}\right)}
$$

which contradicts (3.5). Therefore $\gamma_{1} \neq \gamma_{2}$. If $\gamma_{1}<\gamma_{2}$ then

$$
\frac{1}{a\left(C_{1}\right)}=\rho\left(\widehat{M}_{1}-\left(1-\gamma_{1}\right) \mathbf{e e}^{T}\right)<\rho\left(\widehat{M}_{2}-\left(1-\gamma_{2}\right) \mathbf{e e}^{T}\right)=\frac{1}{a\left(C_{2}\right)} .
$$

Moreover

$$
\frac{1}{a\left(C_{1}\right)}=\rho\left(M_{1}-\gamma_{1} \mathbf{e e}^{T}\right)>\rho\left(M_{2}-\gamma_{2} \mathbf{e e}^{T}\right)=\frac{1}{a\left(C_{2}\right)}
$$

which contradicts (3.6). Consequently, $\gamma_{1}>\gamma_{2}$ and hence

$$
\frac{1}{a\left(C_{1}\right)}=\rho\left(\widehat{M}_{1}-\left(1-\gamma_{1}\right) \mathbf{e e}^{T}\right)>\rho\left(\widehat{M}_{2}-\left(1-\gamma_{2}\right) \mathbf{e e}^{T}\right)=\frac{1}{a\left(C_{2}\right)} .
$$


We have proved that $a\left(C_{1}\right) \leq a\left(C_{2}\right)$ with equality if and only if $C_{1}=C_{2}$.

Observe that $C_{2}$ is isomorphic to

$$
\widetilde{C}_{1}=C\left(p_{2 s}, \ldots, p_{s+1}, \widetilde{p}_{s}, 1, \ldots, 1\right)
$$

and that $C_{3}$ is isomorphic to

$$
\widetilde{C}_{2}=C\left(1, \ldots, 1, \widetilde{p}_{s+1}, \widetilde{p}_{s}, 1, \ldots, 1\right) .
$$

We apply (3.4) to conclude $a\left(\widetilde{C}_{1}\right) \leq a\left(\widetilde{C}_{2}\right)$ with equality if and only if $\widetilde{C}_{1}=\widetilde{C}_{2}$. Therefore $a\left(C_{2}\right) \leq a\left(C_{3}\right)$ with equality if and only if $C_{2}=C_{3}$. By transitivity, $a(C)=$ $a\left(C_{1}\right) \leq a\left(C_{3}\right)=a(\widetilde{C})$ with equality if and only if $C=\widetilde{C}$.

For $d=2 s+1$, let

$$
\mathcal{M}=\left\{C\left(1, \ldots, 1, p_{s}, p_{s+1}, 1, \ldots, 1\right) \in \mathcal{C}: p_{s} \leq p_{s+1}\right\}
$$

Let $p_{s}=a$ and $p_{s+1}=b$. Thus a caterpillar in $\mathcal{M}$ is of the form $C(1, \ldots, 1, a, b, 1, \ldots, 1)$ in which $a+b=n-2 d+4$ and $a \leq b$. For brevity, we write $C(a, b)$ instead of $C(1, \ldots, 1, a, b, 1, \ldots, 1)$.

In order to obtain the caterpillar having the maximum algebraic connectivity in

$$
\mathcal{C}=\left\{C\left(p_{1}, \ldots, p_{s}, p_{s+1}, \ldots, p_{2 s}\right): p_{i} \geq 1 \text { for all } i\right\},
$$

we recall the following theorem.

Theorem 3.5. [14, Theorem 5] Let $d=2 s+1$. The algebraic connectivity of $C(a, b)=C(1, \ldots, 1, a, b, 1, \ldots, 1) \in \mathcal{M}$ is a strictly decreasing function for $1 \leq a \leq$ $\frac{1}{2}(n-2 d+4)$.

We are ready to give the caterpillar with the maximum algebraic connectivity among the caterpillars in $\mathcal{C}$ whenever $d$ is odd.

THEOREM 3.6. If $d=2 s+1$ then among all trees in $\mathcal{C}$ the maximum algebraic connectivity is attained by the caterpillar $C\left(1, \ldots, 1,1, p_{s+1}, 1, \ldots, 1\right)$ where $p_{s+1}=n-$ $2 d+3$.

Proof. Let

$$
C_{1}=C\left(p_{1}, \ldots, p_{s}, p_{s+1}, \ldots, p_{2 s}\right) \in \mathcal{C}
$$

We define

$$
C_{3}=C\left(1, \ldots, 1, \widetilde{p}_{s}, \widetilde{p}_{s+1}, 1, \ldots, 1\right)
$$


where $\widetilde{p}_{s}=\sum_{l=1}^{s} p_{l}-s+1, \widetilde{p}_{s+1}=\sum_{l=s+1}^{2 s} p_{l}-s+1$, and

$$
C_{4}=C(1,1, \ldots, 1, n-2 d+3,1, \ldots, 1) .
$$

Clearly, $C_{3} \in \mathcal{M}$ and $C_{4} \in \mathcal{M}$. From (3.3), $a\left(C_{1}\right) \leq a\left(C_{3}\right)$. If $\widetilde{p}_{s}=1$ then $\widetilde{p}_{s+1}=$ $n-2 d+3$ and thus $a\left(C_{1}\right) \leq a\left(C_{3}\right)=a\left(C_{4}\right)$. If $\widetilde{p}_{s}>1$, from Theorem 3.5, we get $a\left(C_{3}\right)<a\left(C_{4}\right)$ and thus $a\left(C_{1}\right)<a\left(C_{4}\right)$.

\subsection{Minimum algebraic connectivity. Let}

$$
\mathcal{E}=\{C(a, 1, \ldots, 1, b) \in \mathcal{C}: a \leq b, a+b=n-2 d+4\} .
$$

We recall the following theorem.

Theorem 3.7. [14, Theorem 4] The algebraic connectivity of $C(a, 1, \ldots, 1, b) \in \mathcal{E}$ is a strictly decreasing function for $1 \leq a \leq \frac{1}{2}(n-2 d+4)$.

TheOREM 3.8. Among all trees in $\mathcal{C}$ the minimum algebraic connectivity is attained by the caterpillar $C(x, 1,1, \ldots, 1,1, y)$ where $x=\left\lfloor\frac{n-2 d+4}{2}\right\rfloor$ and $y=\left\lceil\frac{n-2 d+4}{2}\right\rceil$.

Proof. Let $C_{1}=C(\mathbf{p}) \in \mathcal{C}$. As in the proof of Lemma 3.4, four cases occur and, in each case, the vertices of the caterpillars are labeled appropriately to compare the entries of the corresponding bottleneck matrices in (3.1) or (3.2).

Case $C_{1}$ is a Type I tree. Let $v_{l}$ be the characteristic vertex of $C_{1}$. We have

$$
\frac{1}{a\left(C_{1}\right)}=\rho\left(M_{1}\right)=\rho\left(\widehat{M}_{1}\right) .
$$

We define $C_{0}=C\left(\widetilde{p}_{1}, 1,1, \ldots, 1,1, \widetilde{p}_{d-1}\right) \in \mathcal{C}$ where

$$
\widetilde{p}_{1}=\sum_{j=1}^{l} p_{j}-(l-1) \text { and } \widetilde{p}_{d-1}=\sum_{j=l+1}^{d-1} p_{j}-(d-l-2) .
$$

We want to prove that $a\left(C_{0}\right) \leq a\left(C_{1}\right)$. We may assume that $C_{0} \neq C_{1}$. Then $p_{t}>1$ for some $2 \leq t \leq d-2$. Suppose that $C_{0}$ is a Type I tree with characteristic vertex $v_{k}$. We have

$$
\frac{1}{a\left(C_{0}\right)}=\rho\left(M_{0}\right)=\rho\left(\widehat{M}_{0}\right) .
$$

If $k=l$ then $M_{0}(p, q)-M_{1}(p, q) \geq 0$ for each $(p, q)$ with strict inequality in at least one entry whenever $2 \leq t \leq l$ or $\widehat{M}_{0}(p, q)-\widehat{M}_{1}(p, q) \geq 0$ for each $(p, q)$ with strict inequality in at least one entry whenever $l+1 \leq t \leq d-2$. Hence

$$
\frac{1}{a\left(C_{0}\right)}=\rho\left(M_{0}\right)>\rho\left(M_{1}\right)=\frac{1}{a\left(C_{1}\right)}
$$


or

$$
\frac{1}{a\left(C_{0}\right)}=\rho\left(\widehat{M}_{0}\right)>\rho\left(\widehat{M}_{1}\right)=\frac{1}{a\left(C_{1}\right)} .
$$

If $k>l$ then $M_{0}(p, q)-M_{1}(p, q)>0$ for each $(p, q)$. Hence

$$
\frac{1}{a\left(C_{0}\right)}=\rho\left(M_{0}\right)>\rho\left(M_{1}\right)=\frac{1}{a\left(C_{1}\right)} \text {. }
$$

If $k<l$ then $\widehat{M}_{0}(p, q)-\widehat{M}_{1}(p, q)>0$ for each $(p, q)$. Hence

$$
\frac{1}{a\left(C_{0}\right)}=\rho\left(\widehat{M}_{0}\right)>\rho\left(\widehat{M}_{1}\right)=\frac{1}{a\left(C_{1}\right)} .
$$

Suppose now that $C_{0}$ is a Type II tree with characteristic vertices $v_{r}$ and $v_{r+1}$. There is $0<\gamma_{0}<1$ such that

$$
\frac{1}{a\left(C_{0}\right)}=\rho\left(M_{0}-\gamma_{0} \mathbf{e e}^{T}\right)=\rho\left(\widehat{M}_{0}-\left(1-\gamma_{0}\right) \mathbf{e e}^{T}\right) .
$$

If $r \geq l$ then $M_{0}(p, q)-M_{1}(p, q) \geq 1$ for each $(p, q)$ and thus

$$
\frac{1}{a\left(C_{0}\right)}=\rho\left(M_{0}-\gamma_{0} \mathbf{e}^{T}\right)>\rho\left(M_{1}\right)=\frac{1}{a\left(C_{1}\right)} .
$$

If $r<l$ then $\widehat{M}_{0}(p, q)-\widehat{M}_{1}(p, q) \geq 1$ for each $(p, q)$ and thus

$$
\frac{1}{a\left(C_{0}\right)}=\rho\left(\widehat{M}_{0}-\left(1-\gamma_{0}\right) \mathbf{e e}^{T}\right)>\rho\left(\widehat{M}_{1}\right)=\frac{1}{a\left(C_{1}\right)} .
$$

We have proved that if $C_{1}$ is a Type $I$ tree and $C_{1} \neq C_{0}$ then $a\left(C_{0}\right)<a\left(C_{1}\right)$.

Case $C_{1}$ is a Type II tree. Let $v_{i}$ and $v_{i+1}$ be the characteristic vertices of $C_{1}$. There is $0<\gamma_{1}<1$ such that

$$
\frac{1}{a\left(C_{1}\right)}=\rho\left(M_{1}-\gamma_{1} \mathbf{e e}^{T}\right)=\rho\left(\widehat{M}_{1}-\left(1-\gamma_{1}\right) \mathbf{e e}^{T}\right) .
$$

In this case, we define $C_{0}=C\left(\widetilde{p}_{1}, 1,1, \ldots, 1,1, \widetilde{p}_{d-1}\right)$ where

$$
\widetilde{p}_{1}=\sum_{j=1}^{i} p_{j}-(i-1) \text { and } \widetilde{p}_{d-1}=\sum_{j=i+1}^{d-1} p_{j}-(d-i-2) \text {. }
$$

We want to prove $a\left(C_{0}\right) \leq a\left(C_{1}\right)$. Suppose $C_{0} \neq C_{1}$. Then $p_{t}>1$ for some $2 \leq t \leq$ $d-2$. Suppose that $C_{0}$ is a Type I tree with characteristic vertex $v_{k}$. We have

$$
\frac{1}{a\left(C_{0}\right)}=\rho\left(M_{0}\right)=\rho\left(\widehat{M}_{0}\right) \text {. }
$$


If $k \leq i$ then $\widehat{M}_{0}(p, q)-\widehat{M}_{1}(p, q) \geq 0$ for each $(p, q)$. Hence

$$
\frac{1}{a\left(C_{0}\right)}=\rho\left(\widehat{M}_{0}\right) \geq \rho\left(\widehat{M}_{1}\right)>\rho\left(\widehat{M}_{1}-\left(1-\gamma_{1}\right) \mathbf{e e}^{T}\right)=\frac{1}{a\left(C_{1}\right)} .
$$

If $k>i$ then $M_{0}(p, q)-M_{1}(p, q) \geq 0$ for each $(p, q)$. Hence

$$
\frac{1}{a\left(C_{0}\right)}=\rho\left(M_{0}\right) \geq \rho\left(M_{1}\right)>\rho\left(M_{1}-\gamma_{1} \mathbf{e e}^{T}\right)=\frac{1}{a\left(C_{1}\right)} .
$$

Suppose now that $C_{0}$ is a Type II tree with characteristic vertices $v_{r}$ and $v_{r+1}$. There is $0<\gamma_{0}<1$ such that

$$
\frac{1}{a\left(C_{0}\right)}=\rho\left(M_{0}-\gamma_{0} \mathbf{e e}^{T}\right)=\rho\left(\widehat{M}_{0}-\left(1-\gamma_{0}\right) \mathbf{e e}^{T}\right) .
$$

If $r<i$ then $\widehat{M}_{0}(p, q)-\widehat{M}_{1}(p, q) \geq 1$ for each $(p, q)$ and thus

$$
\begin{aligned}
\frac{1}{a\left(C_{0}\right)} & =\rho\left(\widehat{M}_{0}-\left(1-\gamma_{0}\right) \mathbf{e e}^{T}\right)>\rho\left(\widehat{M}_{1}\right) \\
& >\rho\left(\widehat{M}_{1}-\left(1-\gamma_{1}\right) \mathbf{e e}^{T}\right)=\frac{1}{a\left(C_{1}\right)} .
\end{aligned}
$$

If $r>i$ then $M_{0}(p, q)-M_{1}(p, q) \geq 1$ for each $(p, q)$ and thus

$$
\begin{aligned}
\frac{1}{a\left(C_{0}\right)} & =\rho\left(M_{0}-\gamma_{0} \mathbf{e} \mathbf{e}^{T}\right)>\rho\left(M_{1}\right) \\
& >\rho\left(M_{1}-\gamma_{1} \mathbf{e} \mathbf{e}^{T}\right)=\frac{1}{a\left(C_{1}\right)} .
\end{aligned}
$$

If $r=i$ and $\gamma_{0}=\gamma_{1}$ then $M_{0}(p, q)-M_{1}(p, q) \geq 0$ for each $(p, q)$ with strict inequality in at least one entry whenever $2 \leq t \leq i$ or $\widehat{M}_{0}(p, q)-\widehat{M}_{1}(p, q) \geq 0$ for each $(p, q)$ with strict inequality in at least one entry whenever $i+1 \leq t \leq d-2$. Hence

$$
\begin{aligned}
\frac{1}{a\left(C_{0}\right)} & =\rho\left(M_{0}-\gamma_{0} \mathbf{e} \mathbf{e}^{T}\right)=\rho\left(M_{0}-\gamma_{1} \mathbf{e} \mathbf{e}^{T}\right) \\
& >\rho\left(M_{1}-\gamma_{1} \mathbf{e} \mathbf{e}^{T}\right)=\frac{1}{a\left(C_{1}\right)} .
\end{aligned}
$$

or

$$
\begin{aligned}
\frac{1}{a\left(C_{0}\right)} & =\rho\left(\widehat{M}_{0}-\left(1-\gamma_{0}\right) \mathbf{e e}^{T}\right)=\rho\left(\widehat{M}_{0}-\left(1-\gamma_{1}\right) \mathbf{e e}^{T}\right) \\
& >\rho\left(\widehat{M}_{1}-\left(1-\gamma_{1}\right) \mathbf{e} \mathbf{e}^{T}\right)=\frac{1}{a\left(C_{1}\right)} .
\end{aligned}
$$

If $r=i$ and $\gamma_{0}<\gamma_{1}$ then $M_{0}(p, q)-M_{1}(p, q) \geq 0$ for each $(p, q)$ and thus

$$
\begin{aligned}
\frac{1}{a\left(C_{0}\right)} & =\rho\left(M_{0}-\gamma_{0} \mathbf{e} \mathbf{e}^{T}\right)>\rho\left(M_{0}-\gamma_{1} \mathbf{e} \mathbf{e}^{T}\right) \\
& \geq \rho\left(M_{1}-\gamma_{1} \mathbf{e} \mathbf{e}^{T}\right)=\frac{1}{a\left(C_{1}\right)} .
\end{aligned}
$$


If $r=i$ and $\gamma_{0}>\gamma_{1}$ then $\widehat{M}_{0}(p, q)-\widehat{M}_{1}(p, q) \geq 0$ for each $(p, q)$ and thus

$$
\begin{aligned}
\frac{1}{a\left(C_{0}\right)} & =\rho\left(\widehat{M}_{0}-\left(1-\gamma_{0}\right) \mathbf{e e}^{T}\right)>\rho\left(\widehat{M_{0}}-\left(1-\gamma_{1}\right) \mathbf{e e}^{T}\right) \\
& \geq \rho\left(\widehat{M}_{1}-\left(1-\gamma_{1}\right) \mathbf{e e}^{T}\right)=\frac{1}{a\left(C_{1}\right)} .
\end{aligned}
$$

We have proved that if $C_{1}$ is a Type $I I$ tree and $C_{1} \neq C_{0}$ then $a\left(C_{0}\right)<a\left(C_{1}\right)$.

Finally, let $\mu$ be the algebraic connectivity of the caterpillar $C(x, 1, \ldots, 1, y)$ in which $x=\left\lfloor\frac{n-2 d+4}{2}\right\rfloor$ and $y=\left\lceil\frac{n-2 d+4}{2}\right\rceil$. Observe that this caterpillar lies in $\mathcal{C}$. We apply Theorem 3.7 , to obtain $\mu \leq a\left(C_{0}\right)$. This completes the proof.

3.4. Extremal algebraic connectivities of symmetric caterpillars. We have

$$
\mathcal{S}=\left\{C(\mathbf{p}) \in \mathcal{C}: p_{j}=p_{d-j}, j=1,2, \ldots,\left\lfloor\frac{d-1}{2}\right\rfloor\right\} .
$$

To find the caterpillars in $\mathcal{S}$ having the maximum and the minimum algebraic connectivity is immediate. In fact, if $d=2 s+2$ then, from Theorem 3.3,

$$
\begin{aligned}
\max \{a(C): C \in \mathcal{S}\} & =a\left(C_{0}\right)=\max \{a(C): C \in \mathcal{C}\} \\
C_{0} & =C\left(1, \ldots, 1, p_{s+1}, 1, \ldots, 1\right) \\
p_{s+1} & =n-2 d+3
\end{aligned}
$$

and if $d=2 s+1$ then, from Lemma 3.4,

$$
\begin{aligned}
\max \{a(C): C \in \mathcal{S}\} & =a\left(C_{0}\right) \\
C_{0} & =C\left(1, \ldots, 1, p_{s}, p_{s+1}, 1, \ldots, 1\right) \\
p_{s} & =p_{s+1}=\frac{1}{2}(n-2 d+4) .
\end{aligned}
$$

If $d=2 s+2$, from Theorem 3.8, for even $n$,

$$
\begin{aligned}
\min \{a(C): C \in \mathcal{S}\} & =a\left(C_{0}\right) \\
C_{0} & =C(x, 1, \ldots, 1,1,1, \ldots, 1, x) \\
x & =\frac{1}{2}(n-2 d+4) .
\end{aligned}
$$

and, for odd $n$,

$$
\begin{aligned}
\min \{a(C): C \in \mathcal{S}\} & =a\left(C_{0}\right) \\
C_{0} & =C(x, 1, \ldots, 1,2,1, \ldots, 1, x) \\
x & =\frac{1}{2}(n-2 d+3) .
\end{aligned}
$$


If $d=2 s+1$, from Theorem 3.8 ,

$$
\begin{aligned}
\min \{a(C): C \in \mathcal{S}\} & =a\left(C_{0}\right) \\
C_{0} & =C(x, 1, \ldots, 1,1, \ldots, 1, x) \\
x & =\frac{1}{2}(n-2 d+3) .
\end{aligned}
$$

3.5. A summary of the extremal algebraic connectivities. Table 1 summarizes the results on the extremal algebraic connectivities on $\mathcal{C}$ and on $\mathcal{S}$ that we have found in this paper. The table includes also the corresponding results for the subclasses $\mathcal{E}$ and $\mathcal{M}$. We observe that these results are immediate consequences of Theorem 3.7 and Theorem 3.5. The columns 2 and 3 give the caterpillars that attain the maximum and the minimum algebraic connectivity, respectively, in the class of caterpillars indicated in column 1.

\begin{tabular}{|c|c|c|}
\hline Class & $\max a(C(\mathbf{p}))$ & $\min a(C(\mathbf{p}))$ \\
\hline \multirow{3}{*}{$\mathcal{C}$} & $d=2 s+1$ or $d=2 s+2$ & $d=2 s+1$ or $d=2 s+2$ \\
& $C\left(1, \ldots, 1, p_{s+1}, 1, \ldots, 1\right)$ & $C(x, 1, \ldots, 1,1, \ldots, 1, y)$ \\
& $p_{s+1}=n-2 d+3$ & $x=\left\lfloor\frac{n-2 d+4}{2}\right\rfloor, y=\left\lceil\frac{n-2 d+4}{2}\right\rceil$ \\
\hline \multirow{E}{*}{ odd or $d$ even } & $d$ odd or $d$ even & $C(x, 1, \ldots, 1, \ldots, 1, y)$ \\
& $C(1,1, \ldots, 1, \ldots, 1, y)$ & $x=\left\lfloor\frac{n-2 d+4}{2}\right\rfloor, y=\left\lceil\frac{n-2 d+4}{2}\right\rceil$ \\
& $y=n-2 d+3$ & $d=2 s+1$ \\
$\mathcal{M}$ & $d=2 s+1$ & $C\left(1, \ldots, 1, p_{s}, p_{s+1}, 1, \ldots, 1\right)$ \\
& $C\left(1, \ldots, 1,1, p_{s+1}, 1, \ldots, 1\right)$ & $p_{s}=\left\lfloor\frac{n-2 d+4}{2}\right\rfloor, p_{s+1}=\left\lceil\frac{n-2 d+4}{2}\right\rceil$ \\
\hline \multirow{3}{*}{$\mathcal{S}$} & $p_{s+1}=n-2 d+3$ & $d=2 s+2$ and $n$ even \\
& $d=2 s+2$ & $C(x, 1, \ldots, 1, \ldots, 1, x)$ \\
& $p_{s+1}=n-2 d+3$ & $x=\frac{1}{2}(n-2 d+4)$ \\
$\mathcal{S}$ & & $C(x, 1, \ldots, 2, \ldots, 1, x)$ \\
& & $x=\frac{1}{2}(n-2 d+3)$ \\
& & $d=2 s+1$ \\
$\mathcal{S}$ & $C\left(1, \ldots, 1, p_{s}, p_{s+1}, 1, \ldots, 1\right)$ & $C(x, 1, \ldots, 1, \ldots, 1, x)$ \\
& $p_{s}=p_{s+1}=\frac{1}{2}(n-2 d+4)$ & $x=\frac{1}{2}(n-2 d+4)$ \\
\hline
\end{tabular}

Table 1: Extremal algebraic connectivities

\section{Characterizing the algebraic connectivity of symmetric caterpillars.} A generalized Bethe tree is a rooted tree in which vertices at the same distance from the root have the same degree. In [13], we characterize completely the Laplacian eigenvalues of the tree $P_{m}\left\{B_{i}\right\}$ obtained from the path $P_{m}$ and $m$ generalized Bethe 
trees $B_{1}, B_{2}, \ldots, B_{m}$ by identifying the root vertex of $B_{i}$ with the $i$-th vertex of $P_{m}$. This is the case for the caterpillar $C(\mathbf{p})$ in which the path is $P_{d-1}$ and the $(d-1)$ generalized Bethe trees are the stars $S_{p_{i}}(1 \leq i \leq d-1)$. Thus we may apply the results in [13] to characterize the algebraic connectivity of $C(\mathbf{p})$. Previously we introduce some notation. Let

$$
\begin{aligned}
& A(x)=\left[\begin{array}{cc}
1 & \sqrt{x} \\
\sqrt{x} & x+1
\end{array}\right] \\
& B(x)=\left[\begin{array}{cc}
1 & \sqrt{x} \\
\sqrt{x} & x+2
\end{array}\right]
\end{aligned}
$$

and

$$
E=\left[\begin{array}{ll}
0 & 0 \\
0 & 1
\end{array}\right]
$$

Applying Theorem 4 of [13] to $C(\mathbf{p})$, one can obtain

THEOREM 4.1. The algebraic connectivity of $C(\mathbf{p})$ is the smallest positive eigenvalue of the $(2 d-2) \times(2 d-2)$ positive semidefinite matrix

$$
M(\mathbf{p})=\left[\begin{array}{ccccc}
A\left(p_{1}\right) & E & & & \\
E & B\left(p_{2}\right) & E & & \\
& \ddots & \ddots & \ddots & \\
& & \ddots & B\left(p_{d-2}\right) & E \\
& & & E & A\left(p_{d-1}\right)
\end{array}\right] .
$$

Next we characterize the algebraic connectivity of a caterpillar in $\mathcal{S}$ as the smallest eigenvalue of a $2 \times 2$ - block tridiagonal matrix of order $2 s \times 2 s$ if $d=2 s+1$ or $d=2 s+2$.

4.1. Case $d=2 s+1$. Let $d=2 s+1$. Let $C(\mathbf{p}) \in \mathcal{S}$. Then

$$
\mathbf{p}=\left[p_{1}, \ldots, p_{s}, p_{s}, \ldots, p_{1}\right]
$$

and the matrix $M(\mathbf{p})$ in (4.1) becomes

$$
M(\mathbf{p})=\left[\begin{array}{ccccc}
A\left(p_{1}\right) & E & & & \\
E & B\left(p_{2}\right) & E & & \\
& \ddots & \ddots & \ddots & \\
& & \ddots & B\left(p_{2}\right) & E \\
& & & E & A\left(p_{1}\right)
\end{array}\right]
$$


of order $4 s \times 4 s$. Let $J$ be the permutation matrix

$$
J=\left[\begin{array}{lllll} 
& & & I \\
& & I & \\
& & & \\
& I & & \\
I & & &
\end{array}\right]
$$

of order $2 s \times 2 s$ in which the $2 \times 2$ blocks in the secondary diagonal are the $2 \times 2$ identity matrix. We may write

$$
M(\mathbf{p})=\left[\begin{array}{cc}
N\left(p_{1}, \ldots, p_{s}\right) & F \\
F & J N\left(p_{1}, \ldots, p_{s}\right) J
\end{array}\right]
$$

where

$$
N\left(p_{1}, \ldots, p_{s}\right)=\left[\begin{array}{ccccc}
A\left(p_{1}\right) & E & & & \\
E & B\left(p_{2}\right) & \ddots & & \\
& \ddots & \ddots & \ddots & \\
& & \ddots & & E \\
& & & E & B\left(p_{s}\right)
\end{array}\right]
$$

and

$$
F=\left[\begin{array}{ccccc}
0 & 0 & & & 0 \\
0 & 0 & \ddots & & \vdots \\
\vdots & \ddots & \ddots & \ddots & \vdots \\
0 & & \ddots & 0 & 0 \\
E & 0 & \cdots & 0 & 0
\end{array}\right]
$$

We claim that $M(\mathbf{p})$ in (4.3) is orthogonally similar to a block diagonal matrix. In fact, let us consider the orthogonal matrix $Q$ of order $4 s \times 4 s$,

$$
Q=\frac{1}{\sqrt{2}}\left[\begin{array}{cc}
I & J \\
I & -J
\end{array}\right]
$$

A computation shows

$$
\begin{aligned}
& Q M(\mathbf{p}) Q^{T} \\
= & {\left[\begin{array}{cc}
R & 0 \\
0 & S
\end{array}\right] }
\end{aligned}
$$


where

$$
R=\left[\begin{array}{ccccc}
A\left(p_{1}\right) & E & & & \\
E & B\left(p_{2}\right) & \ddots & & \\
& \ddots & \ddots & \ddots & \\
& & \ddots & & E \\
& & & E & B\left(p_{s}\right)+E
\end{array}\right]
$$

and

$$
S=\left[\begin{array}{ccccc}
A\left(p_{1}\right) & E & & & \\
E & B\left(p_{2}\right) & \ddots & & \\
& \ddots & \ddots & \ddots & \\
& & \ddots & & E \\
& & & E & B\left(p_{s}\right)-E
\end{array}\right] .
$$

Therefore

$$
\sigma(M(\mathbf{p}))=\sigma(R) \cup \sigma(S) .
$$

Since $\operatorname{det} R=2$ and $\operatorname{det} S=0, R$ is a positive definite matrix and $S$ is positive semidefinite matrix.

We recall the Monotonicity Theorem for eigenvalues of Hermitian matrices [9]:

Theorem 4.2. Let $A, B \in M_{n}$ be Hermitian matrices. Let $C=A+B$. Let

$$
\begin{aligned}
\alpha_{1} & \leq \alpha_{2} \leq \ldots . \leq \alpha_{n} \\
\beta_{1} & \leq \beta_{2} \leq \ldots . \leq \beta_{n} \\
\gamma_{1} & \leq \gamma_{2} \leq \ldots . . \leq \gamma_{n}
\end{aligned}
$$

be the eigenvalues of $A, B$ and $C$ respectively. Then

$$
\begin{array}{ccc}
\alpha_{j}+\beta_{i-j+1} \leq \gamma_{i} & \text { if } & i \geq j \\
\gamma_{i} \leq \alpha_{j}+\beta_{i-j+n} & \text { if } & i \leq j \\
\alpha_{i}+\beta_{1} \leq \gamma_{i} \leq \alpha_{i}+\beta_{n} & & i=1,2, \ldots, n .
\end{array}
$$

TheOrem 4.3. If $d=2 s+1$ and $\mathbf{p}=\left[p_{1}, \ldots, p_{s}, p_{s}, \ldots, p_{1}\right]$ then the algebraic connectivity of the caterpillar $C(\mathbf{p})$ is the smallest eigenvalue of

$$
R=\left[\begin{array}{cccc}
A\left(p_{1}\right) & E & & \\
E & B\left(p_{2}\right) & \ddots & \\
& \ddots & \ddots & E \\
& & E & B\left(p_{s}\right)+E
\end{array}\right]
$$


Proof. We have

$$
R=S+T
$$

where all the entries of $T$ are zeros except for $T(2 s, 2 s)=2$. Let

$$
\begin{aligned}
\gamma_{1} & \leq \gamma_{2} \leq \ldots \leq \gamma_{2 s} \\
0 & =\alpha_{1} \leq \alpha_{2} \leq \ldots \leq \alpha_{2 s} \\
0 & =\beta_{1}=\ldots=\beta_{2 s-1}<\beta_{2}=2
\end{aligned}
$$

be the eigenvalues of $R, S$ and $T$ respectively. From (4.5)

$$
\sigma(M(\mathbf{p}))=\left\{\gamma_{1}, \gamma_{2}, \ldots, \gamma_{2 s}\right\} \cup\left\{0, \alpha_{2}, \ldots, \alpha_{2 s}\right\}
$$

From Theorem 4.2

$$
\gamma_{i} \leq \alpha_{j}+\beta_{i-j+2 s} \text { for } i \leq j
$$

In particular, for $i=1$ and $j=2$, we have $\gamma_{1} \leq a_{2}+\beta_{2 s-1}=\alpha_{2}+0=\alpha_{2}$. This shows that the smallest positive eigenvalue of $R$ is the smallest positive eigenvalue of $M(\mathbf{p})$. This fact and Theorem 4.1 complete the proof. $\square$

EXAMPLE 4.4. Consider the symmetric caterpillar

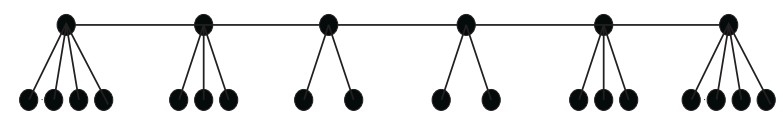

We have $\mathbf{p}=\left[\begin{array}{llllll}4 & 3 & 2 & 2 & 3 & 4\end{array}\right]$ and its algebraic connectivity is the smallest eigenvalue of

$$
R=\left[\begin{array}{cccccc}
1 & 2 & 0 & 0 & 0 & 0 \\
2 & 5 & 0 & 1 & 0 & 0 \\
0 & 0 & 1 & \sqrt{3} & 0 & 0 \\
0 & 1 & \sqrt{3} & 5 & 0 & 1 \\
0 & 0 & 0 & 0 & 1 & \sqrt{2} \\
0 & 0 & 0 & 1 & \sqrt{2} & 5
\end{array}\right]
$$

4.2. Case $d=2 s+2$. Let $d=2 s+2$. Let $C(\mathbf{p}) \in \mathcal{S}$. Then

$$
\mathbf{p}=\left[p_{1}, p_{2}, \ldots, p_{s}, p_{s+1}, p_{s}, \ldots, p_{2}, p_{1}\right]
$$


and the matrix $M(\mathbf{p})$ in (4.1) becomes

$$
M(\mathbf{p})=\left[\begin{array}{ccc}
N\left(p_{1}, \ldots, p_{s}\right) & {\left[\begin{array}{c}
0 \\
E
\end{array}\right]} & 0 \\
{\left[\begin{array}{ll}
0 & E
\end{array}\right]} & {\left[\begin{array}{cc}
1 & \sqrt{p_{s+1}} \\
\sqrt{p_{s+1}} & p_{s+1}+2
\end{array}\right]} & {\left[\begin{array}{ll}
E & 0
\end{array}\right]} \\
0 & {\left[\begin{array}{c}
E \\
0
\end{array}\right]} & J N\left(p_{1}, \ldots, p_{s}\right) J
\end{array}\right]
$$

where $N\left(p_{1}, \ldots, p_{s}\right)$ and $J$ are as in (4.4) and (4.2) respectively. Let us consider the orthogonal matrix $Q$ of order $(4 s+2) \times(4 s+2)$

$$
Q=\frac{1}{\sqrt{2}}\left[\begin{array}{ccc}
I & 0 & J \\
0 & \sqrt{2} I & 0 \\
I & 0 & -J
\end{array}\right] .
$$

A computation shows

$$
\begin{aligned}
& Q M(\mathbf{p}) Q^{T} \\
& =\left[\begin{array}{ccc}
N\left(p_{1}, \ldots, p_{s}\right) & {\left[\begin{array}{c}
0 \\
\sqrt{2} E
\end{array}\right]} & 0 \\
{\left[\begin{array}{cc}
0 & \sqrt{2} E
\end{array}\right]} & {\left[\begin{array}{cc}
1 & \sqrt{p_{s+1}} \\
\sqrt{p_{s+1}} & p_{s+1}+2
\end{array}\right]} & 0 \\
0 & 0 & N\left(p_{1}, \ldots, p_{s}\right)
\end{array}\right] .
\end{aligned}
$$

Hence

$$
\sigma(M(\mathbf{p}))=\sigma(U) \cup \sigma(N)
$$

where

$$
U=\left[\begin{array}{cc}
N\left(p_{1}, \ldots, p_{s}\right) & {\left[\begin{array}{c}
0 \\
\sqrt{2} E
\end{array}\right]} \\
{\left[\begin{array}{ll}
0 & \sqrt{2} E
\end{array}\right]} & {\left[\begin{array}{cc}
1 & \sqrt{p_{s+1}} \\
\sqrt{p_{s+1}} & p_{s+1}+2
\end{array}\right]}
\end{array}\right] .
$$

Since $\operatorname{det} U=0$ and $\operatorname{det} N=1, U$ is a positive semidefinite matrix and $N$ is a positive definite matrix.

The proof of the following theorem as well as the following example are given in [1, Theorem 8 and Example 3]. However, for completeness, they are also given here. 
Theorem 4.5. If $d=2 s+2$ and $\mathbf{p}=\left[p_{1}, \ldots, p_{s}, p_{s+1}, p_{s}, \ldots, p_{1}\right]$ then the algebraic connectivity of $C(\mathbf{p})$ is the smallest eigenvalue of

$$
N=\left[\begin{array}{cccc}
A\left(p_{1}\right) & E & & \\
E & B\left(p_{2}\right) & \ddots & \\
& \ddots & \ddots & E \\
& & E & B\left(p_{s}\right)
\end{array}\right] .
$$

Proof. We know that the smallest positive eigenvalue of $M(\mathbf{p})$ is the algebraic connectivity of $C(\mathbf{p})$. From (4.6) the eigenvalues of $M(\mathbf{p})$ are the eigenvalues of $U$ and $N$. Let $U_{1}$ the principal submatrix of $U$ obtained by deleting its last row and its last column. That is

$$
U_{1}=\left[\begin{array}{cc}
N & 0 \\
0 & 1
\end{array}\right]
$$

We have $\operatorname{det} N=1$. Then at least one eigenvalue of $N$ is strictly less than 1 . By the eigenvalue interlacing property for Hermitian matrices, the eigenvalues of $N$ and the eigenvalue 1 interlace the eigenvalues of $U$. These facts all together imply that the smallest eigenvalue of $N$ is the smallest positive eigenvalue of $M(\mathbf{p})$. This completes the proof. $\square$

EXAMPLE 4.6. The algebraic connectivity of the caterpillar

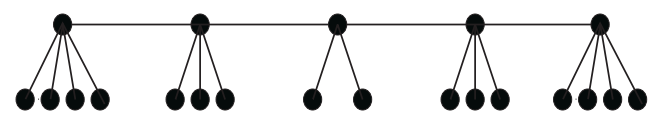

is the smallest eigenvalue of

$$
N=\left[\begin{array}{cccc}
1 & \sqrt{4} & 0 & 0 \\
\sqrt{4} & 5 & 0 & 1 \\
0 & 0 & 1 & \sqrt{3} \\
0 & 1 & \sqrt{3} & 5
\end{array}\right]
$$

Acknowledgment. The authors wish to thank the referee for the comments which led to an improved version of the paper. 


\section{REFERENCES}

[1] N. Abreu, O. Rojo, and C. Justel. Algebraic connectivity for subclasses of caterpillars. Appl. Anal. Discrete Math., in press (http://pefmath.etf.rs/accepted/rrad850.pdf).

[2] T. Biyikoğlu and J. Leydold. Algebraic connectivity and degree sequences of trees. Linear Algebra Appl., 430:811-817, 2009.

[3] N. M. M. de Abreu. Old and new results on algebraic connectivity of graphs. Linear Algebra Appl., 423:53-73, 2007.

[4] S. Fallat and S. Kirkland. Extremizing algebraic connectivity subject to graph theoretic constraints. Electron. J. Linear Algebra, 3:48-74, 1998.

[5] M. Fiedler. Algebraic connectivity of graphs, Czechoslovak Math. J., 23:298-305, 1973.

[6] M. Fiedler. A property of eigenvectors of nonnegative symmetric matrices and its applications to graph theory. Czechoslovak Math. J., 25:619-633, 1975.

[7] R. Grone and R. Merris. Ordering trees by algebraic connectivity. Graphs Combin., 6:229-237, 1990.

[8] R. Grone, R. Merris, and V.S. Sunder. The Laplacian spectrum of a graph. SIAM J. Matrix Anal. Appl., 11:218-238, 1990.

[9] Y. Ikebe, T. Inagaki, and S. Miyamoto. The monotonicity theorem, Cauchy's interlace theorem and the Courant-Fischer theorem. Amer. Math. Monthly, 94:352-354, 1987.

[10] S. Kirkland, M. Neumann, and B. L. Shader. Characteristic vertices of weighted trees via Perron values. Linear and Multilinear Algebra, 40:311-325, 1996.

[11] R. Merris. Characteristic vertices of trees. Linear and Multinear Algebra, 22:115-131, 1987.

[12] R. Merris. Laplacian matrices of graphs: A survey. Linear Algebra Appl., 197/198:143-176, 1994.

[13] O. Rojo and L. Medina. Spectra of generalized Bethe trees attached to a path. Linear Algebra Appl., 430:483-503, 2009.

[14] O. Rojo, L. Medina, N.M.M. de Abreu, and C. Justel. On the algebraic connectivity of some of caterpillars: A sharp upper bound and a total ordering. Linear Algebra Appl., 432:586-605, 2010.

[15] J-Y. Shao, J-M. Guo, and H-Y. Shan. The ordering of trees and connected graphs by algebraic connectivity. Linear Algebra Appl., 428:1421-1438, 2008.

[16] R. S. Varga. Matrix Iterative Analysis. Prentice-Hall, Inc., Englewood Cliffs, New Jersey, 1962.

[17] X-Y. Yuan, J-Y. Shao and L. Zhang, The six classes of trees with the largest algebraic connectivity. Discrete Appl. Math., 156:757-769, 2008.

[18] L. Zhang and Y. Liu. Ordering trees with nearly perfect matchings by algebraic connectivity. Chin. Ann. Math. Ser. B, 29:71-84, 2008.

[19] X-D. Zhang. Ordering trees with algebraic connectivity and diameter. Linear Algebra Appl., 427:301-312, 2007. 